\title{
Nonlinear dynamics of large-amplitude dust acoustic shocks and solitary pulses in dusty plasmas
}

\author{
P. K. Shukla ${ }^{1,2, *}$ and B. Eliasson ${ }^{1}$ \\ ${ }^{1}$ International Centre for Advanced Studies in Physical Sciences \& Institute for Theoretical Physics, Faculty of Physics \& Astronomy, \\ Ruhr University Bochum, D-44780 Bochum, Germany \\ ${ }^{2}$ Department of Mechanical and Aerospace Engineering \& Centre for Energy Research, University of California, San Diego, La Jolla, \\ California 92093, USA
}

(Received 25 May 2012; published 15 October 2012)

\begin{abstract}
We present a fully nonlinear theory for dust acoustic (DA) shocks and DA solitary pulses in a strongly coupled dusty plasma, which have been recently observed experimentally by Heinrich et al. [Phys. Rev. Lett. 103, 115002 (2009)], Teng et al. [Phys. Rev. Lett. 103, 245005 (2009)], and Bandyopadhyay et al. [Phys. Rev. Lett. 101, 065006 (2008)]. For this purpose, we use a generalized hydrodynamic model for the strongly coupled dust grains, accounting for arbitrary large-amplitude dust number density compressions and potential distributions associated with fully nonlinear nonstationary DA waves. Time-dependent numerical solutions of our nonlinear model compare favorably well with the recent experimental works (mentioned above) that have reported the formation of large-amplitude nonstationary DA shocks and DA solitary pulses in low-temperature dusty plasma discharges.
\end{abstract}

DOI: 10.1103/PhysRevE.86.046402

PACS number(s): 52.27.Lw, 52.35.Fp, 52.35.Tc

\section{INTRODUCTION}

Charged dust grains and dusty plasmas [1-7] are ubiquitous in astrophysical environments (e.g., interstellar media, molecular dusty clouds, star forming clouds, and supernovae such as the Eagle Nebula), in planetary ring systems [1,8] (e.g., the spokes in Saturn's rings recorded by the Voyager spacecraft cameras), and in our solar system (e.g., interplanetary dust particles produced by comets), as well as near the Sun's and Earth's atmospheres (e.g., the mesospheric and ionospheric regions). Charged dust particles are naturally formed in industrial processing of nanotechnology and in magnetic fusion reactors.

It is well known that charging of a neutral dust particle occurs due to a variety of physical processes $[9,10]$, including the collection of electrons from the background plasma, photo emissions, triboelectric effects, etc. In the remote past, it was shown by Wuerker et al. [11] that an ensemble of electrically charged iron and aluminum particles having diameters of a few microns can be confined by three-dimensional focusing forces of alternating and static electric fields and the Coulomb repulsion, leading eventually to the formation of crystallized arrays of ions and aluminum dust particles, which can be melted and reformed. However, a dusty plasma is usually composed of electrons, positive ions, negative or positive dust grains, and neutral atoms. When the interaction potential energy (which is equal to $Z_{d}^{2} e^{2} / d$, where $Z_{d}$ is the dust charge state, $e$ is the magnitude of the electron charge, and $d$ is the interdust grain distance or the Wigner-Seitz radius) between two neighboring dust grains is much larger (smaller) than the dust kinetic energy $k_{B} T_{d}$, where $k_{B}$ is the Boltzmann constant and $T_{d}$ is the dust temperature, the dusty plasma is in a strongly (weakly) coupled state. Following the charged particle condensation idea [12] of a one-component strongly correlated electron system, Ikezi [13] postulated

*profshukla@yahoo.de the solidification of charged dust particles when the dusty plasma $\Gamma=Z_{d}^{2} e^{2} \exp \left(-d / \lambda_{D}\right) / d k_{B} T_{d}$ exceeds 172 , taking into account the plasma screening effect, where $\lambda_{D}$ is the plasma Debye radius [2]. Such values of $\Gamma$ can be achieved in low-temperature laboratory discharges at room temperatures owing to the large $Z_{d}$ acquired by a micron-size dust grain by absorbing electrons from the background plasma. There are also Monte Carlo and molecular dynamics simulations that accurately depict different states of ordered dust structures [14-16] when dust grains are repelling each other according to the Yukawa or Debye-Hückel force. The phase diagram for $\Gamma_{s}$ against $\kappa[=$ (average interdust grain spacing)/(dusty plasma Debye radius)] indeed reveals dust solid face-centered-cubic, dust solid body-centered-cubic, and dust fluid phases for a set of $\Gamma_{s}$ and $\kappa$ values, as given in Ref. [16], where an empirical scaling for dust crystal melting is also given.

The formation of dust Coulomb crystals and ordered dust structures has been observed in the sheath region of many laboratory experiments [17-20], where charged dust grains are kept together due to confining electrostatic potentials in a plasma sheath. However, robust ordered dust structures may also be formed due to attractive forces [2] between negative dust grains associated with ion focusing and ion wake fields $[21,22]$ in a dusty plasma sheath with streaming ions, shadowing forces due to collisions with ions [23,24], and overlapping Debye spheres [25] and dipole-dipole interactions [26-28]. The alignment of charged dust grains in an assembly due to the attractive force associated with ion focusing and ion wake field effects has been experimentally observed [29]. Furthermore, the collective behavior of dusty plasmas involving an ensemble of charged dust grains was recognized through the prediction of the dust acoustic wave (DAW) by Shukla [30], who suggested the existence of the nonlinear DAW in the presence of Boltzmann distributed electrons and ions and massive, charged dust particles. This idea was then worked out in a paper [31] on the DAW. It must be stressed that there does not exist a counterpart of the DAW in an electron-ion plasma without charged dust grains since the 
DAW is supported by the dust particle inertia and the restoring force comes from the pressures of the inertialess hot electron and ions. Thus, similar to the Alfvén wave in a magnetized plasma, the DAW is of fundamental importance in laboratory and space plasmas physics. The DAW is usually excited by an ion streaming instability and has a frequency much smaller than the dusty plasma frequency, extending into the infrasonic frequency range. Low-frequency (of the order of $10 \mathrm{~Hz}$ ) dust acoustic (DA) fluctuations were observed in the experiment of Chu et al. [17] and have since been observed in many laboratory experiments worldwide [2,6,17,32-35] and also in the Earth's ionosphere [36].

Ichimaru et al. [37] further extended the theory of strong coupling and viscosity coefficients for a high-density onecomponent electron plasma. Berkovsky [38] developed a generalized hydrodynamic model for plasmas with strongly coupled ions and degenerate electrons and used it to investigate the linear properties of modified ion-acoustic waves. A similar theory was developed for strongly correlated dust grains in dusty plasmas by Kaw and Sen [39], who presented a generalized viscoelastic hydrodynamic model for strongly correlated dust grains and investigated the linear properties of dust acoustic waves, especially the low-frequency longitudinal and transverse modes in a strongly coupled dusty plasma. The latter model has also been extended to the weakly nonlinear regime [40] to study the propagation of small-amplitude nonlinear dust acoustic waves in a strongly coupled dusty plasma.

However, recently a number of laboratory experiments [41-45] have reported observations of nonlinear DAWs in the form of extremely large-amplitude DA shocks [41-43] and DA solitary pulses $[35,44,45]$ at kinetic levels. Physically, the large-amplitude DA shocks are formed when nonlinearities in plasmas balance the DAW dissipation caused by the dust fluid viscosity coming from dust grain correlations in strongly coupled dusty plasmas, while DA solitary pulses arise in the collisionless regime due to the balance between the harmonic generation nonlinearities and the DAW dispersion. We are unaware of theories for arbitrary large-amplitude nonlinear, nonstationary DA shocks and DA solitary pulses in dusty plasmas with dust correlations. It should be stressed that small-amplitude theories for DA shocks and DA solitary pulses based on the Burgers [46], Korteweg-de Vries $(\mathrm{KdV})$, and $\mathrm{KdV}$-Burgers equations [40] are not suitable for explaining observations [33,42-45] that report anomalously high (up to $40 \%$ and beyond) dust density compressions. A large-amplitude theory of Eliasson and Shukla [47] for a collisionless dusty plasma explains well the DAW steepening and nonlinear wave speed $[42,43]$, but is unable to predict the shock width observed in the experiments.

In this paper we present a fully nonlinear, nonstationary unified theory for arbitrary large-amplitude DA shocks and DA solitary pulses in a dusty plasma, taking into account the effects of strong coupling between charged dust grains, the nonlinear polarization force acting on charged dust grains due to thermal ions that shield negative dust grains, collisions between charged dust grains and neutrals, dust correlations decay rate, the dust fluid shear and bulk viscosities, etc. This gives a more complete picture of various nonideal effects in dusty plasmas, and we are thus able to provide a comparison between our new nonstationary and fully nonlinear theory with the recent laboratory observations of DA shocks and DA solitary pulses [42-45]. Neglected are effects due to attractive forces (ion focusing, wake fields, etc.) between dust grains, which may affect the equation of state and transport coefficients of the system. These effects, however, are either small or depend on the moment transfer of streaming ions, which we do not consider here.

\section{MATHEMATICAL MODEL}

We consider a dusty plasma composed of inertialess electrons and ions, as well as strongly correlated negatively charged micron-sized dust particles of uniform sizes, in the presence of large-amplitude ultralow-frequency DA waves, with $\omega \ll v_{e n}$ and $\nu_{i n} \ll k^{2} V_{T e, T i}^{2} / \omega$, where $\omega$ is the wave frequency, $v_{e n}\left(v_{i n}\right)$ is the electron- (ion)-neutral collision frequency, $k$ is the wave number, and $V_{T e}\left(V_{T i}\right)$ is the electron (ion) thermal speed. Both electrons and ions follow the Boltzmann law since they can be considered inertialess on the time scale of the DAW period and henceforth rapidly thermalize under the action of collisions. Thus the electron and ion number densities are, respectively, $n_{e}=n_{e 0} \exp \left(e \phi / k_{B} T_{e}\right)$ and $n_{i}=n_{i 0} \exp \left(-e \phi / k_{B} T_{i}\right)$, where $n_{e 0}$ and $n_{i 0}$ are the unperturbed electron and ion number densities, respectively, $e$ is the magnitude of the electron charge, $\phi$ is the electrostatic potential, $k_{B}$ is the Boltzmann constant, and $T_{e}\left(T_{i}\right)$ is the electron (ion) temperature. At equilibrium, we have the quasineutrality condition $n_{i 0}=n_{e 0}+Z_{d} n_{d 0}$, where $Z_{d}$ is the average number of electrons residing on a dust grain and $n_{d 0}$ is the unperturbed dust number density.

The dust particle dynamics associated with fully nonlinear, nonstationary DAWs in a strongly coupled dusty plasma is governed by the generalized hydrodynamic equations composed of the dust continuity equation $\left(\partial n_{d} / \partial t\right)+\nabla \cdot\left(n_{d} \mathbf{v}_{d}\right)=0$ and the generalized dust momentum equation

$$
\begin{gathered}
\left(1+\tau_{r} \frac{d}{d t}\right)\left[\frac{d \mathbf{v}_{d}}{d t}+v_{d} \mathbf{v}_{d}-\frac{Z_{d} e}{m_{d}} \nabla \phi+\frac{Z_{d} e R}{m_{d}}\left(\frac{n_{i}}{n_{i 0}}\right)^{1 / 2} \nabla \phi\right. \\
\left.+\frac{k_{B} T_{d}}{\rho_{d}} \nabla\left(\mu_{d} n_{d}\right)\right]=\frac{\eta}{\rho_{d}} \nabla^{2} \mathbf{v}_{d}+\frac{\xi+\frac{\eta}{3}}{\rho_{d}} \nabla\left(\nabla \cdot \mathbf{v}_{d}\right),
\end{gathered}
$$

taking into account finite-amplitude convective and pressure nonlinearities [47], nonlinear ion polarization force, strong dust coupling effects [37-39,48], and dust neutral collisions [49]. Here $d / d t=(\partial t / \partial t)+\mathbf{v}_{d} \cdot \nabla$ is the total time derivative; $n_{d}$ and $\mathbf{v}_{d}$ are the dust number density and dust fluid velocity, respectively; $m_{d}$ is the dust mass; $\rho_{d}=n_{d} m_{d}$ is the dust mass density; $R=Z_{d} e^{2} / 4 k_{B} T_{i} \lambda_{D i}$ is a parameter determining the effect of the polarization force [50], which reduces the phase speed of the DAW, arising from interactions between thermal ions and negative dust grains; and $\mu_{d} n_{d} k_{B} T_{d} \equiv P_{d}$ is the effective dust thermal pressure for a one-component plasma [39], where $\mu_{d}=1+(1 / 3) u(\Gamma)+(\Gamma / 9) \partial u(\Gamma) / \partial \Gamma$ is the compressibility, $\Gamma=Z_{d}^{2} e^{2} / d k_{B} T_{d}$ is the ratio between the dust Coulomb and dust thermal energies, $d=\left(3 / 4 \pi n_{d 0}\right)^{1 / 3}$ is the Wigner-Seitz dust grain separation distance, and $u(\Gamma)$ is a measure of the excess internal energy of the system, which reads [51,52] $u(\Gamma) \simeq-(\sqrt{3} / 2) \Gamma^{3 / 2}$ for $\Gamma \leqslant 1$ (viz., a liquidlike state) and $u(\Gamma)=-0.80 \Gamma+0.95 \Gamma^{1 / 4}+0.19 \Gamma^{-1 / 4}-0.81$ in a 
range $1<\Gamma<200$. Furthermore, the effective dusty plasma Debye radius $\lambda_{D}=\lambda_{D e} \lambda_{D i} /\left(\lambda_{D e}^{2}+\lambda_{D i}^{2}\right)^{1 / 2}$, where $\lambda_{D e}=\left(k_{B} T_{e} / 4 \pi n_{e 0} e^{2}\right)^{1 / 2}$ and $\lambda_{D i}=\left(k_{B} T_{i} / 4 \pi n_{i 0} e^{2}\right)^{1 / 2}$ are the ion and electron Debye radii, respectively. The dust-neutral collision frequency is given by the Epstein formula [49] $v_{d n}=$ $(8 / 3) \sqrt{2 \pi} m_{n} n_{n} r_{d}^{2} v_{T n} / m_{d}$, where $m_{n}$ is the neutral mass, $n_{n}$ is the neutral number density, $r_{d}$ is the dust grain radius, $V_{T n}=\left(k_{B} T_{n} / m_{n}\right)^{1 / 2}$ is the neutral thermal speed, and $T_{n}$ is the neutral gas temperature. The viscoelastic properties of the dust fluids are characterized by the dust correlation relaxation time $[37,38] \tau_{r}=\left[(\xi+4 \eta / 3) / n_{d 0} T_{d}\right] /\left[1-\mu_{d}+4 u(\Gamma) / 15\right]$, involving the shear and bulk viscosities $\eta$ and $\xi$, respectively. There are various approaches for calculating $\eta$ and $\xi$, which are widely discussed in the literature [52]. The DA wave potential $\phi$ is obtained from Poisson's equation $\nabla^{2} \phi=4 \pi e\left(n_{e}-n_{i}+Z_{d} n_{d}\right)$, taking into account the dispersive effect due to the departure from quasineutrality. The ion drag force [53-55] acting on a dust grain has been neglected in Eq. (1), which is justified since the ions are assumed to follow the Boltzmann distribution with no ion momentum flow. However, in a dusty plasmas with large dust particles and equilibrium ion flows, there can be an instability with a growth rate much smaller than the DAW frequency [2].

\section{ONE-DIMENSIONAL QUASISTATIONARY SHOCKS AND SOLITARY WAVES}

Let us now consider the simplest problem of onedimensional nonlinear DAWs propagating along the $x$ axis in a Cartesian coordinate system. We define the dimensionless variables $N=n_{d} / n_{d 0}, U=\hat{\mathbf{x}} \cdot \mathbf{v}_{d} / C_{d}$, and $\Phi=$ $e \phi / k_{B} T_{i}$, where $C_{d}=\omega_{p d} \lambda_{D}$ is the dust acoustic speed, $\omega_{p d}=\left(4 \pi n_{d 0} Z_{d}^{2} e^{2} / m_{d 0}\right)^{1 / 2}$ is the dust plasma frequency, and $\hat{\mathbf{x}}$ the unit vector along the $x$ axis. We then have the dust continuity equation

$$
\frac{D N}{D T}+N \frac{\partial U}{\partial X}=0
$$

the generalized viscoelastic dust momentum equation

$$
\begin{aligned}
& \left(1+a \frac{D}{D T}\right)\left[\frac{D U}{D T}+v U-[1-R \exp (-\Phi / 2)] \frac{\gamma}{P} \frac{\partial \Phi}{\partial X}\right. \\
& \left.+T_{0} \frac{\partial \ln N}{\partial X}\right]-\frac{\beta}{\Lambda} \frac{\partial^{2} U}{\partial X^{2}}=0,
\end{aligned}
$$

and Poisson's equation

$$
\gamma \frac{\partial^{2} \Phi}{\partial X^{2}}=(1-P) \exp (\tau \Phi)-\exp (-\Phi)+P N,
$$

where $a=\omega_{p d} \tau_{r}, v=v_{d n} / \omega_{p d}, D / D T=\partial / \partial T+U \partial / \partial X$, $T=\omega_{p d} t, \quad X=x / \lambda_{D}, \quad \Lambda=\lambda_{D}^{2} / d^{2}, \quad \beta=(\xi+4 \eta / 3) /$ $m_{d} n_{d 0} \omega_{p d} d^{2}$ (typical values [37] of $\beta$ are roughly 1.04, 0.08 , and 0.3 for $\Gamma=1,10$, and 160, respectively), $T_{0}=\mu_{d} T_{d} \gamma / Z_{d} T_{i} P, \quad \gamma=1+\tau(1-P), \quad P=Z_{d} n_{d 0} / n_{i 0}$, and $\tau=T_{i} / T_{e}$. We are assuming here that the constant parameter $P$ is given for a set of experiments; however, it has been experimentally shown [9] that $Z_{d}$ is typically reduced for closely packed $\left(d<\lambda_{D}\right)$ dust grains. This effect, which can be important at high dust number densities, will be neglected here for simplicity. Furthermore, the dust charge fluctuation effect has been neglected since the dust charging time period $v_{1}^{-1}$ is usually much shorter than the time period for the formation of nonlinear DAWs we are concerned with [56] and the fugacity parameter $\mathcal{F}=4 \pi n_{d 0} \lambda_{D i}^{2} r_{d} v_{2} / v_{1}\left(1+n_{e 0} T_{i} / n_{i 0} T_{e}\right)$ is smaller than 1 , with the expressions for $v_{1}$ and $v_{2}$ given in Refs. [2,56].

In a stationary frame such that all physical variables depend only on $\zeta=X-M T$ with $M=U / C_{d}$, where $U$ is the constant speed of the nonlinear DA waves. We have $U=$ $M(N-1) / N$, so that the dust momentum equation (3) reads

$$
\begin{gathered}
\left(1-\frac{a M}{N} \frac{\partial}{\partial \zeta}\right)\left[\frac{M^{2}}{2} \frac{\partial}{\partial \zeta}\left(\frac{1}{N^{2}}\right)+v M \frac{N-1}{N}\right. \\
\left.-[1-R \exp (-\Phi / 2)] \frac{\gamma}{P} \frac{\partial \Phi}{\partial \zeta}+T_{0} \frac{\partial \ln N}{\partial \zeta}\right] \\
+\frac{\beta M}{\Lambda} \frac{\partial^{2}}{\partial \zeta^{2}}\left(\frac{1}{N}\right)=0,
\end{gathered}
$$

which couples with Poisson's equation

$$
\gamma \frac{\partial^{2} \Phi}{\partial \zeta^{2}}=(1-P) \exp (\tau \Phi)-\exp (-\Phi)+P N .
$$

Quasistationary DA shock waves exist only for $v=0$, when the dust-neutral collisions can be neglected. Furthermore, it is possible to derive a simple condition for the DA shock wave amplitudes depending on other parameters when the relaxation time for dust grain correlations is much smaller than the dust plasma period. Hence, for $a=v=0$, Eq. (5) can be integrated once to obtain

$$
\begin{aligned}
& \frac{M^{2}}{2}\left(\frac{1}{N^{2}}-1\right)-\frac{\gamma}{P} \Phi+\frac{2 \gamma R}{P}[1-\exp (-\Phi / 2)]+T_{0} \ln N \\
& \quad+\frac{\beta M}{\Lambda} \frac{\partial}{\partial \zeta}\left(\frac{1}{N}\right)=0
\end{aligned}
$$

where we have used the boundary conditions $N=1$, $\Phi=0$, and $\partial / \partial \xi=0$ at $\zeta=+\infty$. The DA shock amplitude at $\zeta=-\infty$, where $\partial / \partial \xi=0, \quad N=N_{\text {shock }}>1$, and $\Phi=\Phi_{\text {shock }}<0$, is now obtained from Eq. (7) as

$$
\begin{aligned}
& \frac{M^{2}}{2}\left(\frac{1}{N_{\text {shock }}^{2}}-1\right)-\frac{\gamma}{P} \Phi_{\text {shock }}+\frac{2 \gamma R}{P}\left[1-\exp \left(-\Phi_{\text {shock }} / 2\right)\right] \\
& \quad+T_{0} \ln N_{\text {shock }}=0
\end{aligned}
$$

while Eq. (6) yields

$$
N_{\text {shock }}=\frac{\exp \left(-\Phi_{\text {shock }}\right)-(1-P) \exp \left(\tau \Phi_{\text {shock }}\right)}{P} .
$$

Using Eq. (9) we can eliminate $N_{\text {shock }}$ from Eq. (8) to obtain $M$ as a function of the shock wave potential $\Phi_{\text {shock }}$ for the parameters $R, T_{0}, P$, and $\tau$. The term proportional to $\beta / \Lambda$ in Eq. (7) works to smooth the shock front, but does not influence the shock amplitude. The DA shocks are associated with a positive jump of the dust number density $N_{\text {shock }}>1$ and a decrease of the potential $\Phi_{\text {shock }}<0$ for $M>C_{a}$, where $C_{a}=$ $\left(1-R+T_{0}\right)^{1 / 2}$ is the linear DAW speed in the long-wave limit $\partial / \partial \zeta=0$. Hence the DA shocks are propagating with super-DA speeds in comparison with the upstream plasma.

\section{COMPARISON WITH EXPERIMENTS}

In Figs. 1 and 2 we have used the plasma parameters of Refs. [42,43] to study the nonlinear dynamics and the 

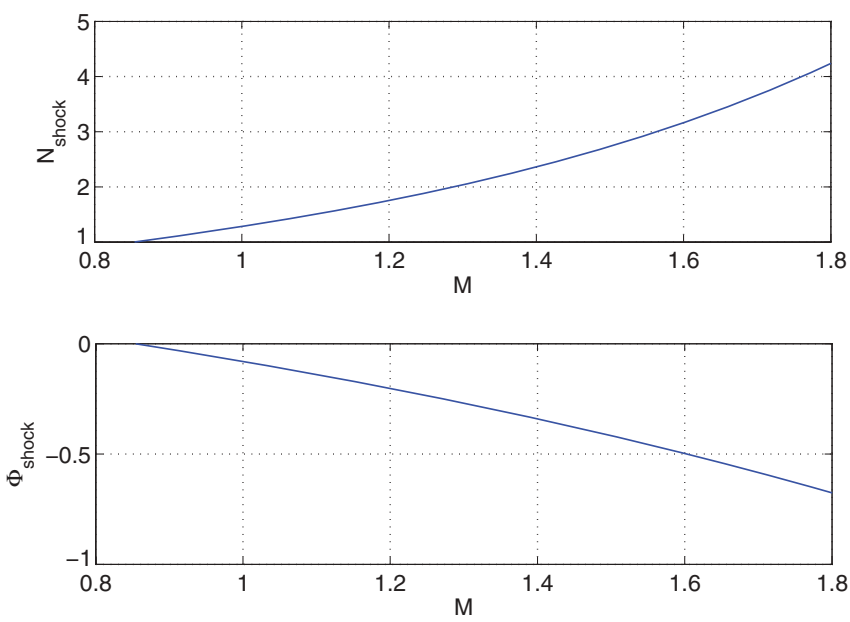

FIG. 1. (Color online) The DA shock potential and associated dust number density as a function of $M$ for $P=0.3, \tau=0.012$, $R=0.28$, and $T_{0}=0.01$. The DA shock potential is negative for increasing dust number density. The amplitudes increase with the increase of $M$.

formation of shocks involving large-amplitude DA pulses. The parameters of the experiment [42] are $n_{i}=2 \times$ $10^{14} \mathrm{~m}^{-3}, T_{i}=0.03 \mathrm{eV}, T_{e}=2.5 \mathrm{eV}, Z_{d}=2 \times 10^{3}, n_{d}=$ $3 \times 10^{10} \mathrm{~m}^{-3}, \quad m_{d}=10^{-15} \mathrm{~kg}$, and $r_{d}=0.5 \mu \mathrm{m}$, giving $\omega_{p d}=590 \mathrm{~s}^{-1}, \lambda_{D} \approx 85 \mu \mathrm{m}, C_{d}=50 \mathrm{~mm} / \mathrm{s}$, and $d \approx 2 \times$ $10^{-4} \mathrm{~m}$. The used gas (argon, $m_{n}=3.6 \times 10^{-29} \mathrm{~kg}$ ) at a pressure of $13 \mathrm{~Pa}$ and temperature $T_{n}=0.03 \mathrm{eV}$ gives a neutral number density $n_{n}=3 \times 10^{21} \mathrm{~m}^{-3}$ and a dust-neutral collision frequency $v_{d n} \approx 1 \mathrm{~s}^{-1}$. It was observed in the experiment [42] that a large-amplitude dust density pulse selfsteepened and formed a shocklike structure, which propagated with a mean speed of about $75 \mathrm{~mm} / \mathrm{s}$, somewhat higher than the estimated dust acoustic speed. For the given parameters, we have $\Lambda=0.18, R=0.28, P=0.3$, and $\tau=0.012$. The normalized dust-neutral collision frequency $v \approx 3 \times 10^{-3}$ is

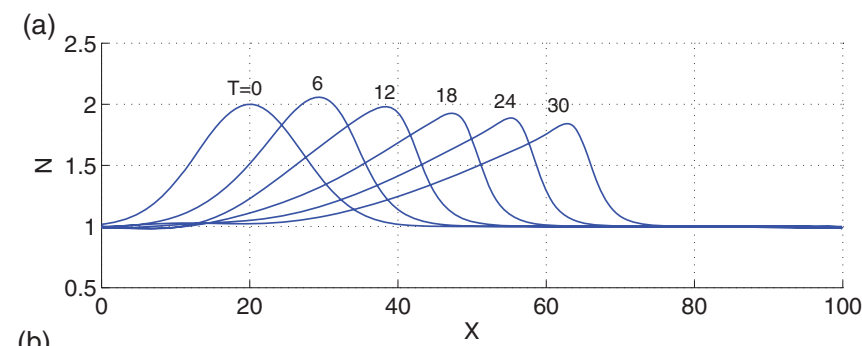

(b)

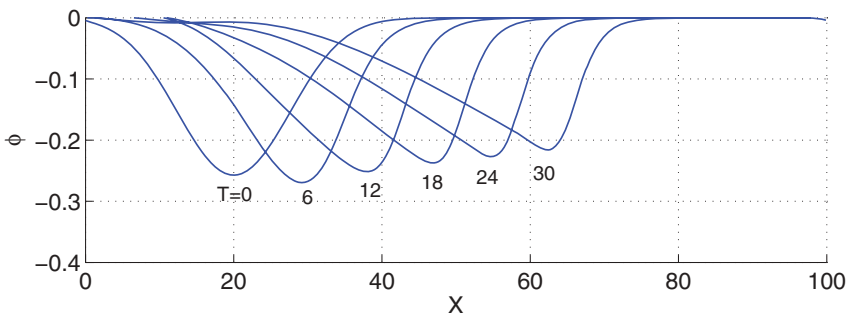

FIG. 2. (Color online) Time and space evolution of (a) the dust number density and (b) the DA wave potential for $a=0.01, \beta=$ $0.15, \Lambda=0.18, v=0.002, P=0.3, R=0.28, T_{0}=0.01$, and $\tau=$ 0.012 , corresponding to the plasma parameters of Refs. [42,43]. quite small, while the dust fluid viscosity due to strong dust coupling effects is more prominent. We choose $\beta=0.15$, which is compatible with the experimental $\Gamma \gtrsim 1$. In addition, we choose $a=T_{0}=0.01$. Figure 1 displays $M$ as a function of dust number density and associated potential, obtained from Eqs. (8) and (9). In the small-amplitude limit, viz., $N_{\text {shock }} \rightarrow 1$ and $\Phi_{\text {shock }} \rightarrow 0$, we have $M \rightarrow C_{a} \approx 0.85$. The DA shock speed $M$ increases with increasing DA shock wave amplitudes, with an increase of the dust density and an associated negative potential. Figure 2 shows a simulation of the time-dependent system of equations (5)-(8). As initial conditions we used $N=1+\exp \left[-(X-20)^{2} / 100\right]$ and $U=0.7 \exp \left[-(X-20)^{2} / 100\right]$. The profiles of the dust number density and DAW potential in Fig. 2 show that the initial DA pulse steepens and a monotonic DA shock is formed, similar to the one in Fig. 5 of Ref. [42]. The largeamplitude $(100 \%)$ dust density perturbations are associated with a negative potential $\Phi \approx-0.25$. The average speed of the DA density pulse is $M \approx 1.4$, in good agreement with Fig. 1 for $N_{\text {shock }} \approx 2, M \approx 1.3$, and $\phi_{\text {shock }} \approx-0.25$. We found that monotonic (oscillatory) DA shocks exist for $\beta \gtrsim \Lambda(\beta \lesssim \Lambda)$ and solitary waves in the limit $\beta \ll \Lambda$. In dimensional units, the simulated nonlinear wave speed is about $70 \mathrm{~mm} / \mathrm{s}$, which is close to the experimental value in Ref. [42].

We next turn to laboratory observations of large-amplitude localized DA solitary pulses in weakly collisional plasma discharges. Teng et al. [45] and Chang et al. [35] observed the formation of large-amplitude localized dust density structures, driven by a flow of ions towards the bottom of the plasma discharge. From the given parameters $[35,45] n_{e}=10^{9} \mathrm{~cm}^{-3}$, $T_{e}=4 \mathrm{eV}, n_{i}=1.2 \times 10^{9} \mathrm{~cm}^{-3}, T_{i}=0.05 \mathrm{eV}, Z_{d}=5000$, $n_{d} \approx 3.7 \times 10^{4} \mathrm{~cm}^{-3}$ (interdust distance about $0.3 \mathrm{~mm}$ ), and $m_{d}=6.9 \times 10^{-11} \mathrm{~g}$, we have $\omega_{p d}=200 \mathrm{~s}^{-1}, \lambda_{D} \approx 45 \mu \mathrm{m}$, and $C_{d}=9 \mathrm{~mm} / \mathrm{s}$. The observed nonlinear DA solitary pulses in Fig. 1(c) of Ref. [45] had a periodicity of about $2 \mathrm{~mm}$, a mean speed of about $45 \mathrm{~mm} / \mathrm{s}$, and a crest width (measured at the height of $N$ where $N=1$ ) in the range $0.4-0.5 \mathrm{~mm}$, with higher-amplitude pulses having smaller widths. We believe that there are some uncertainties in the plasma parameters that could explain the relatively low value of $C_{d}$ compared to the observed wave speed: Increasing the values of $T_{i}$ to $0.10 \mathrm{eV}$ and using the dust charging equation [e.g., Eq. (11) of Shukla and Eliasson [6]], we obtain $Z_{d}=13800$ for $n_{i}=$ $10^{9} \mathrm{~cm}^{-3}$ and $n_{e}=4.9 \times 10^{8} \mathrm{~cm}^{-3}$, giving $\omega_{p d}=540 \mathrm{~s}^{-1}$, $\lambda_{D}=76 \mu \mathrm{m}$, and $C_{d}=41 \mathrm{~mm} / \mathrm{s}$, which is compatible with the experiment. Using these parameters in our model, we have $P=0.51$ and $\tau=0.025$, which we use in the simulation of the time-dependent system of equations (2)-(4). The results are displayed in Fig. 3. We drive the DAW resonantly by an external force of the form $F=-0.01 \sin [2 \pi(X-T) / L]-$ $0.001 \sin [2 \pi(X-T) / 5 L]$, added to the terms in the large square brackets in Eq. (3), where $L=26.3$ is the observed wave periodicity $(2 \mathrm{~mm})$ normalized by $\lambda_{D}$. The result in Fig. 3 shows almost periodic wave trains that develop into narrow peaks, very similar to the ones observed by Teng et al. [45], with density maxima about twice the ambient density and a typical width of about 4-5 Debye radii corresponding to about $0.3-0.4 \mathrm{~mm}$. These spikes may be interpreted as driven largeamplitude solitary DAW structures due to a balance between 


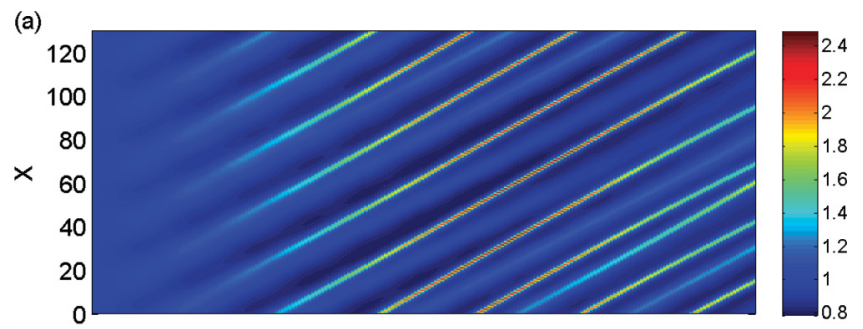

(b)

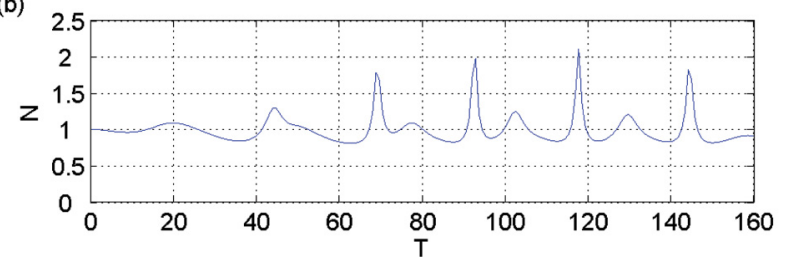

FIG. 3. (Color online) (a) Time and space evolution of the dust number density for $a=\beta=v=R=T_{0}=0, P=0.51$, and $\tau=$ 0.025 . (b) Time variation of $N$ at $X=0$. The driven DAW develops into spiky solitary DAW structures similar to those observed by Teng et al. [45].

the harmonic generation nonlinearities of the system and the dispersion provided by the departure from the quasineutrality condition.

Bandyopadhyay et al. [44] studied how the speeds of DA solitary pulses depend on their amplitudes. The experimental plasma parameters were $n_{i}=7 \times 10^{13} \mathrm{~m}^{-3}, T_{i}=$ $0.3 \mathrm{eV}, T_{e}=8 \mathrm{eV}, n_{d}=10^{10} \mathrm{~m}^{-3}, Z_{d}=3 \times 10^{3}$, and $m_{d}=$ $10^{-13} \mathrm{~kg}$, giving $\omega_{p d}=51 \mathrm{~s}^{-1}, \lambda_{D}=490 \mu \mathrm{m}$, and $C_{d}=$ $25 \mathrm{~mm} / \mathrm{s}$, which corresponds to $P=0.43$ and $\tau=0.038$ in our model. The DA solitary pulses propagated with superdust acoustic speeds, increasing with increasing amplitudes. In

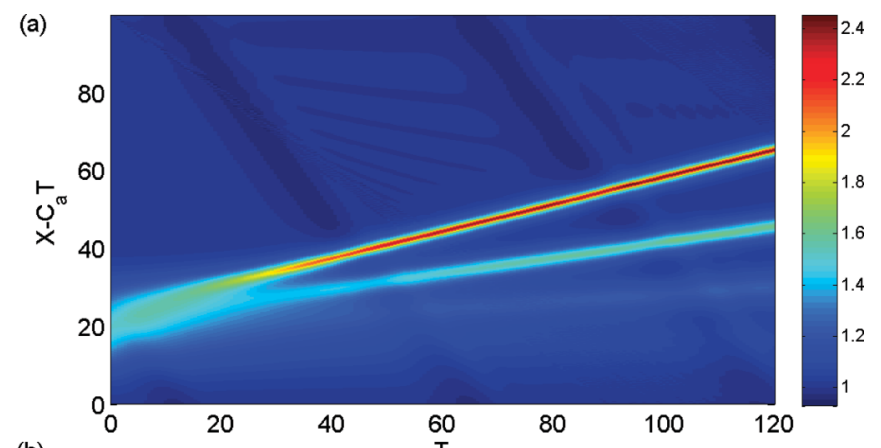

(b)

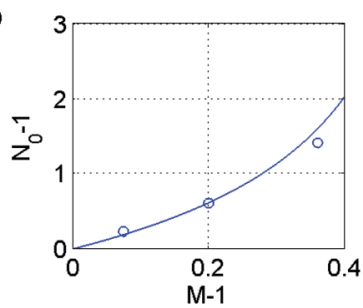

FIG. 4. (Color online) (a) Time and space evolution of the dust number density $N$ for $a=\beta=v=R=T_{0}=0, P=0.43$, and $\tau=0.038$. The initial broad pulse breaks up into three separate DA solitary pulses propagating with superacoustic speed, similar to those observed by Bandyopadhyay et al. [44]. (b) Comparison between the soliton amplitude obtained numerically (circles) and the theoretical amplitude $N_{0}$ (solid line).
Fig. 1(b) of Ref. [44] a pulse of $100 \%$ density amplitude propagates about $8 \times 10^{-3} \mathrm{~m}$ in $0.24 \mathrm{~s}$, giving a mean speed of $v_{d} \approx 0.033 \mathrm{~m} / \mathrm{s}$, which corresponds to $M=v_{d} / C_{d}=1.33$. Figure 4 shows a simulation result, where the initial condition consists of a wide pulse of the form $N=1+0.5 \exp [-(X-$ $\left.20)^{2} / 100\right], \quad U=0.5 \exp \left[-(X-20)^{2} / 100\right]$. The DA pulse breaks up into three DA solitary wave structures propagating with the superdust acoustic speed $M>C_{a}=1$. Smallbut finite-amplitude DA solitary pulses have the density profile $N=1+N_{0} \operatorname{sech}^{2}\left(C_{0}^{1 / 2} \zeta / 2\right)$ and the associated DAW potential $\Phi=-\left(M^{2} P N_{0} / \gamma\right) \operatorname{sech}^{2}\left(C_{0}^{1 / 2} \zeta / 2\right)$, where $N_{0}=$ $3 C_{0} \gamma / 2 B M^{2} P$ is the amplitude, $C_{0}=1-1 / M^{2}$, and $B=$ $(1 / 2 \gamma)\left[(1-P) \tau^{2}-1+3 \gamma^{2} / M^{4} P\right]$. Figure 4(b) exhibits that the numerically obtained amplitudes of the three DA solitary pulses compare favorably well with the theoretical amplitude $N_{0}$. A pulse with a density amplitude of $N_{0}=2$ would have a speed of $M \approx 1.3$, which is also in good agreement with the experiment of Ref. [44].

\section{CONCLUSION}

In summary, we have presented a fully nonlinear, nonstationary unified theory for arbitrary large-amplitude DA shocks and DA solitary pulses in a strongly coupled dusty plasma. Our nonlinear theory is based on the Boltzmann distributed inertialess warm electrons and ions, Poisson's equation, the dust continuity equation, and the generalized viscoelastic dust momentum equation for strongly correlated charged dust grains. The governing nonlinear equations have been numerically solved to obtain the profiles of nonlinear DA waves, including the development of the DA shocks and DA solitary pulses. A comparison between our simulation results and recent experimental observations [42,43] of the DA shocks in laboratory dusty plasma discharges reveals very good agreement with respect to the nonlinear DA wave speeds and DA shock wave smoothing due to strong coupling effects between charged dust particles. From the width of the DA shocks, one may, as suggested by Heinrich et al. [42], infer the dust fluid viscosity. Furthermore, our simulation results of large-amplitude DA solitary pulses also compare favorably well with the observations of Bandyopadhyay et al. [44] and Teng et al. [45]. Future experiments of nonlinear DAWs with higher-precision measurements of the plasma parameters would be very valuable to benchmark the theoretical model. In closing, we stress that our fully nonlinear unified theory for DA shocks and DA solitary pulses remains valid for a dusty plasma with a weak magnetic field (of the order of $100 \mathrm{G}$ ) since the latter is unable to magnetize micron-sized charged dust particles and would not affect the trajectories of electrons and ions that follow the Boltzmann law on the spatiotemporal scales of our interest. A weak magnetic field just provides confinement for the electrons, which are coupled with ions and negative dust grains through the space charge electric field of the DAW.

\section{ACKNOWLEDGMENT}

This research was supported by the Deutsche Forschungsgemeinschaft, Bonn, through Project No. SH21/3-2 of the Research Unit 1048. 
[1] C. K. Goertz, Rev. Geophys. 27, 271 (1989).

[2] P. K. Shukla and A. A. Mamun, Introduction to Dusty Plasma Physics (Institute of Physics, Bristol, 2002).

[3] D. A. Mendis, Plasma Sources Sci. Technol. 11, A219 (2002).

[4] S. V. Vladimirov, K. Ostrikov, and A. A. Samarian, Physics and Applications of Complex Plasmas (Imperial College Press, London, 2005).

[5] V. E. Fortov, A. G. Khrapak, S. A. Khrapak, V. I. Molotkov, and O. F. Petrov, Phys. Usp. 47, 447 (2004); A. V. Ivlev, S. A Khrapak, A. G. Khrapak, and G. E. Morfill, Phys. Rep. 421, 1 (2005).

[6] P. K. Shukla and B. Eliasson, Rev. Mod. Phys. 81, 25 (2009); G. E. Morfill and A. Ivlev, ibid. 81, 1353 (2009).

[7] Complex and Dusty Plasmas: From Laboratory to Space, edited by V. E. Fortov and G. E. Morfill (CRC, London, 2010).

[8] M. Horányi, T. W. Hartquist, O. Havnes, D. A. Mendis, and G. E. Morfill, Rev. Geophys. 42, RG4002 (2004).

[9] A. Barkan, N. D’Angelo, and R. L. Merlino, Phys. Rev. Lett. 73, 3093 (1994).

[10] D. A. Mendis and M. Rosenberg, Annu. Rev. Astronom. Astrophys. 32, 419 (1994).

[11] R. F. Wuerker, H. Shelton, and R. V. Langmuir, J. Appl. Phys. 30, 342 (1959).

[12] S. Ichimaru, Rev. Mod. Phys. 54, 1017 (1982).

[13] H. Ikezi, Phys. Fluids 29, 1764 (1986).

[14] S. Hamaguchi, R. T. Farouki, and D. H. E. Dubin, Phys. Rev. E 56, 4671 (1997); O. S. Vaulina and S. A. Khrapak, JETP 90, 287 (2000).

[15] V. Nosenko, S. K. Zhdanov, A. V. Ivlev, C. A. Knapek, and G. E. Morfill, Phys. Rev. Lett. 103, 015001 (2009).

[16] A. V. Ivlev, G. E. Morfill, and S. A. Khrapak, in Complex and Dusty Plasmas: From Laboratory to Space (Ref. [7]), pp. 240242.

[17] J. H. Chu, J. B. Du, and Lin I, J. Phys. D 27, 296 (1994); Lin I, C.-S. Cherna, J. H. Chu, J.-M. Liu, and J. B. Du, Physica A 205, 443 (1994).

[18] J. H. Chu and Lin I, Phys. Rev. Lett. 72, 4009 (1994).

[19] H. Thomas, G. E. Morfill, V. Demmel, J. Goree, B. Feuerbacher, and D. Möhlmann, Phys. Rev. Lett. 73, 652 (1994).

[20] Y. Hayasi and K. Tachibana, Jpn. J. Appl. Phys. 33, L804 (1994); Y. Hayashi, Phys. Rev. Lett. 83, 4764 (1999).

[21] S. V. Vladimirov and M. Nambu, Phys. Rev. E 52, R2172 (1995).

[22] P. K. Shukla and N. N. Rao, Phys. Plasmas 3, 1770 (1996).

[23] A. M. Ignatov, Plasma Phys. Rep. 22, 585 (1996); V. N. Tsytovich, Y. K. Khodataev, and R. Bingham, Comments Plasma Phys. Control. Fusion 17, 249 (1996); V. N. Tsytovich, Phys. Usp. 40, 53 (1997).

[24] M. Lampe, G. Joyce, G. Ganguli, and V. Gavrishchaka, Phys. Plasmas 7, 3851 (2000).

[25] D. P. Resendes, J. T. Mendonça, and P. K. Shukla, Phys. Lett. A 239, 181 (1998).

[26] H. C. Lee, D. Y. Chen, and B. Rosenstein, Phys. Rev. E 56, 4596 (1997).

[27] U. Mohideen, H. U. Rahman, M. A. Smith, M. Rosenberg, and D. A. Mendis, Phys. Rev. Lett. 81, 349 (1998).

[28] D. D. Tskhakaya and P. K. Shukla, JETP 98, 53 (2004).
[29] K. Takahashi, T. Oishi, K.-i. Shimomai, Y. Hayashi, and S. Nishino, Phys. Rev. E 58, 7805 (1998); G. A. Hebner and M. E. Riley, ibid. 69, 026405 (2004); M. Kroll, J. Schablinski, D. Block, and A. Piel, Phys. Plasmas 17, 013702 (2010).

[30] P. K. Shukla, in Proceedings of the First Capri Workshop on Dusty Plasmas, Capri, 1989, edited by C. Nappi (Consiglio Nazionale delle Aicerche Instituto di Cibernetica, Arco Felice, Napoli, 1989), pp. 38-39.

[31] N. N. Rao, P. K. Shukla, and M. Y. Yu, Planet. Space Sci. 38, 543 (1990).

[32] A. Barkan, R. L. Merlino, and N. D’Angelo, Phys. Plasmas 2, 3563 (1995).

[33] V. I. Molotkov, A. P. Nefedov, V. M. Torchinskii, V. E. Fortov, and A. G. Khrapak, JETP 89, 477 (1999); V. E. Fortov, A. G. Khrapak, S. A. Khrapak, V. I. Molotkov, A. P. Nefedov, O. F. Petrov, and V. M. Torchinsky, Phys. Plasmas 7, 1374 (2000).

[34] H. R. Prabhakara and V. L. Tanna, Phys. Plasmas 3, 3176 (1996).

[35] M.-C. Chang, L.-W. Teng, and Lin I, Phys. Rev. E 85, 046410 (2012).

[36] S. I. Kopnin, S. I. Popel, and M. Y. Yu, Phys. Plasmas 16, 063705 (2009).

[37] S. Ichimaru and S. Tanaka, Phys. Rev. Lett. 56, 2815 (1986); S. Ichimaru, H. Iyetomi, and S. Tanaka, Phys. Rep. 149, 91 (1987).

[38] M. A. Berkovsky, Phys. Lett. A 166, 365 (1992).

[39] P. K. Kaw and A. Sen, Phys. Plasmas 5, 3552 (1998).

[40] B. M. Veeresha, S. K. Tiwari, A. Sen, P. K. Kaw, and A. Das, Phys. Rev. E 81, 036407 (2010).

[41] V. E. Fortov, O. F. Petrov, V. I. Molotkov, M. Y. Poustylnik, V. M. Torchinsky, V. N. Naumkin, and A. G. Khrapak, Phys. Rev. E 71, 036413 (2005).

[42] J. Heinrich, S. H. Kim, and R. L. Merlino, Phys. Rev. Lett. 103, 115002 (2009).

[43] R. L. Merlino, J. R. Heinrich, S.-H. Hyun, and J. K. Meyer, Phys. Plasmas 19, 057301 (2012).

[44] P. Bandyopadhyay, G. Prasad, A. Sen, and P. K. Kaw, Phys. Rev. Lett. 101, 065006 (2008).

[45] L. W. Teng, M. C. Chang, Y. P. Tseng, and Lin I, Phys. Rev. Lett. 103, 245005 (2009).

[46] A. A. Mamun and R. A. Cairns, Phys. Rev. E 79, 055401(R) (2009).

[47] B. E. Eliasson and P. K. Shukla, Phys. Rev. E 69, 067401 (2004).

[48] Y. I. Frenkel, Kinetic Theory of Liquid (Clarendon, Oxford, 1946).

[49] M. F. Baines, L. P. Williams, and A. S. Asebiomo, Mon. Not. R. Astron. Soc. 130, 63 (1965).

[50] S. A. Khrapak, A. V. Ivlev, V. V. Yaroshenko, and G. E. Morfill, Phys. Rev. Lett. 102, 245004 (2009).

[51] R. Abe, Prog. Theor. Phys. 21, 475 (1959).

[52] W. L. Slattery, G. D. Doolen, and H. E. DeWitt, Phys. Rev. A 21, 2087 (1980).

[53] M. S. Barnes, J. H. Keller, J. C. Forster, J. A. ONeill, and D. K. Coultas, Phys. Rev. Lett. 68, 313 (1992).

[54] S. A. Khrapak, A. V. Ivlev, S. K. Zhdanov, and G. E. Morfill, Phys. Plasmas 12, 042308 (2005).

[55] I. H. Hutchinson, Plasma Phys. Controlled Fusion 48, 185 (2006).

[56] P. K. Shukla, Phys. Plasmas 19, 083704 (2012). 